\title{
Topology in Biology: Singularities and Surgery Transformations in Metazoan Development and Evolution
}

\author{
Valeria V. Isaeva ${ }^{1,2}$, Nickolay V. Kasyanov ${ }^{3}$, Eugene V. Presnov ${ }^{4}$ \\ ${ }^{1}$ A.N. Severtsov Institute of Ecology and Evolution of the Russian Academy of Science, Moscow, Russia \\ ${ }^{2}$ A.V. Zhirmunsky Institute of Marine Biology, Far East Branch of Russian Academy of Sciences, Vladivostok, Russia \\ ${ }^{3}$ Institute of Theory and History of Architecture and Town Planning, Moscow, Russia \\ ${ }^{4}$ The Volcani Center, Gilat, Israel \\ Email: vv isaeva@mail.ru, kas_nv@mail.ru, epresnov@agri.gov.il
}

Received 24 July 2014; revised 20 August 2014; accepted 7 September 2014

Copyright (C) 2014 by authors and Scientific Research Publishing Inc.

This work is licensed under the Creative Commons Attribution International License (CC BY).

http://creativecommons.org/licenses/by/4.0/

c) (i) Open Access

\section{Abstract}

The review presents a topological description and interpretation (analysis) of some events in metazoan development and evolution through the use of well-known mathematical concepts and theorems (using topological approach). It is the topological language that can provide strict and adequate description of various phenomena in developmental and evolutionary transformations. Topological singularities inevitably arising and transforming during early development destroy the preexisting pattern of symmetry. The symmetry breaking of preexisting spatial pattern plays a critical role in biological morphogenesis in development and evolution. Some events of early development are interpreted in terms of symmetry breakdown and related to well-known mathematical theorems. A topological inevitability of some developmental events through the use of classical topological concepts is discussed. The topological approach makes it possible to consider the succession of spherical surgeries, which change the topological genus of an animal body surface. We model the biological shape as a set of smooth, closed, oriented surfaces-membrane or epithelial layers. Membrane and epithelial surfaces are boundary layers, interfaces between a living structure and its environment, ensuring metabolism. Toroid forms as well as fractal structures in metazoans can be considered as functionally optimized biological design and attractors in biological morphogenesis. The epithelial surface is an interface between the internal medium of an organism and the outside environmental medium; topological and fractal transformations during metazoan evolution and development increase this interface, ensuring better adaptation of organism to the environment. Fractal structures as well as toroid forms can be considered as a functionally optimized design in Metazoa. Topological methodology reveals a certain set of topological rules constraining and directing biological morphogenesis during evolution and development. 


\section{Keywords}

\section{Topological Surgery, Singularity, Symmetry Breakdown, Evolutionary Biology, Embryology}

\section{Introduction}

The living organisms inhabit and develop in the real physical space and are organized according to the properties of this space. There are general principles in physics, geometry and topology certainly is applied to many situations in biology [1]. Spatial organization of living systems has a dynamical character and includes such discontinuities as topological surgeries and symmetry breaking. Topological language and models can provide strict and adequate description and modeling of biological objects and events since topology operates with the most general properties of spaces as mathematical subjects. Topological modifications of metazoan body patterns in development and evolution are discrete, qualitative steps in biological morphogenesis. Discrete character of biological morphogenesis involves qualitative topological discontinuities, topological catastrophes [2]-[4].

Topological analysis of DNA structure and functions showed the power of the topological methodology see [5] [6]. Following R. Thom [2]-[4] we applied topological concepts and some theorems to describe and interpret developmental and evolutionary form dynamics. In our publications we introduce the topological language into descriptions of biological development at different levels of organization [7]-[15].

The topological approach to description of biological forms and morphogenetic processes has become a more common practice covering the fields of evolutionary and developmental biology, animal behavior and self-organization, neurology, metagenomic biology and biomedicine [9] [10] [14]-[19]. Topological studies by molecular biologists became the field of biochemical topology e.g. [5] [6]. Accordingly, the topological analysis of metazoan transformation can be called topological biology or biological topology [14] [15].

The topological methodology makes it possible to consider as a whole the succession of shape transformations during metazoan development and evolution. Topological approach reveals also a certain set of topological rules constraining and directing biological morphogenesis during evolution and development.

\section{Topological Singularities and Symmetry Transformations in Early Development}

Because an animal body is formed from the spherical egg cell, symmetry breakdown is one of the fundamental processes of development. Symmetry breaking and symmetry propagation are fundamental processes in biological morphogenesis, in metazoan evolution and development [1] [20].

In most metazoans, the animal-vegetal (anterior-posterior) axis of the egg and future embryo is established during oogenesis and usually depends on the position of oocyte with respect to its cellular and non-cellular surrounding. The first symmetry breakdown is associated with the emergence of morphological and functional gradients along animal-vegetal axis of an oocyte. It was shown that spatial anisotropy in distribution of gene products in ooplasm determines the polarity of egg and embryo specifying polar axes of future organism [21]. Cytoskeleton rearrangements break cell symmetry [22]. Actin and tubulin were shown to polymerize forming cytoskeletal actin filaments and microtubules that define the shape of a cell, its polarity and migration, intracellular transport, and the mitotic spindle forming during division. Both the actin and microtubule cytoskeletal elements work together to convert this initial asymmetry into a global cell polarity, for example, in polarization of Drosophila oocytes [20] [22]. A polarized cellular architecture is fundamental to formation of embryonic axes [20]. Egg cytoskeleton functions as a global morphogenetic determinant, which directs and maintains the anisotropy of molecular information in the ooplasm so determining axis polarity of an egg and a future organism [14]. Different organisms use different mechanisms to establish polarity but all rely mainly on the actin and microtubule cytoskeletons. Radially symmetrical arrays of microtubules in animal cells are often called asters, and the presence of such aster breaks the cell symmetry [22]. In egg cells, the aster as visualized topological singularity localizes near animal egg pole.

The axial polarity of an egg is manifested in transcellular ion flows, which generates the extracellular electric field as signals and effectors of the egg polarity [23] (Figure 1(a)).

Egg cell polarization as the emergence of the topological singularity (or singularities) of the vector field on a sphere is inevitable according to the Poincaré-Hopf theorem: "For any smooth vector field given on two-dimen- 
sional sphere $S^{2}$, a singular point of the field exists” [9]-[12]. The vector field on the sphere has at least one singularity, i.e. the point where the value of the field becomes zero (Figure 2(a)). This singular point may be either animal or vegetal pole, or both setting the animal-vegetal differences. This singularity breaks the spherical symmetry of the egg, polarizing the egg sphere [9] [10] [12] [14].

In some animals, at least in chordates, the second symmetry breaking takes place following gamete contact and fusion; this symmetry breaking process involves the asymmetrical flow of cortical actin networks [22]. Sperm penetration in an egg triggers the whole cascade of phenomena, resulting in the wave of calcium release, the electric current of egg activation (Figure 1(b)) and a rearrangement of cortical structure [24] [25]. In chordates, besides preexisting animal egg pole, a new singularity emerges following penetration of a spermium and its spermaster that determines dorso-ventral axis of the egg and future animal see [15] [22] [26]. So the local order of sperm penetration is connected to the integral order of the egg, zygote and developing organism. This symmetry breaking process involves the ooplasmic segregation as a flow of cortical actin networks and displacement of cortical egg components [14] [26]. Ooplasmic segregation creates the spatial-temporal vector field of the cortical displacement, and a singularity of this field is the fixed point of ooplasmic segregation. We consider ooplasmic segregation as a mapping a ball into itself [9] [10] [12]. According to Brouwer's theorem on fixed point, any continuous mapping of the ball into itself has a fixed point. During ooplasmic segregation, subcellular components of an egg are moving with respect to a fixed, stationary point in the course of continuous deformation. The local order of ooplasmic segregation is connected to integral order of the zygote and developing organism [9] [12]. Egg symmetry transformations provide the most important developmental information determining axial pattern of future animal.

In a cleaving zygote, the spatial organization of blastomeres is a genetically determined feature of taxonomic value. The cleavage results in a pattern of cellular contacts on the surface of an embryo that is a discrete cell

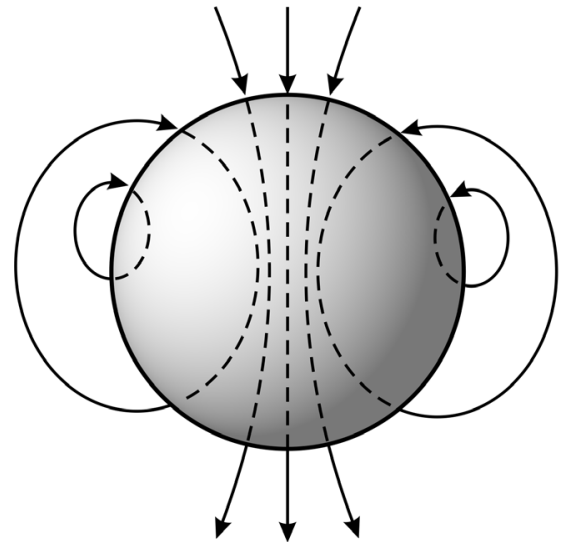

(a)

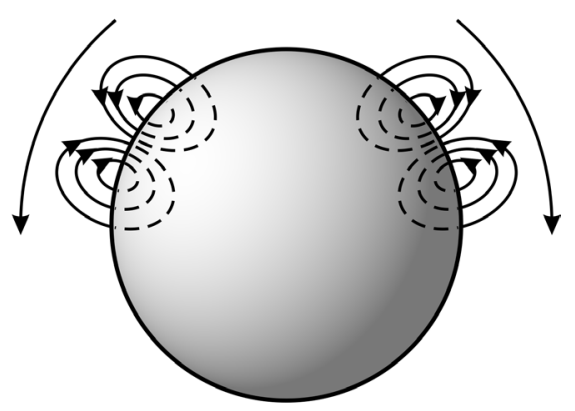

(b)

Figure 1. Electric currents in eggs of the frog Xenopus laevis: (a) The electric field in an oocyte (after [23], modified); (b) The wave of the activation current following sperm penetration (after [27] modified).

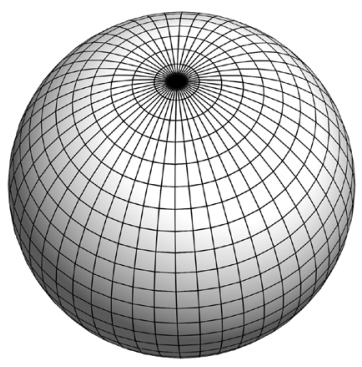

(a)

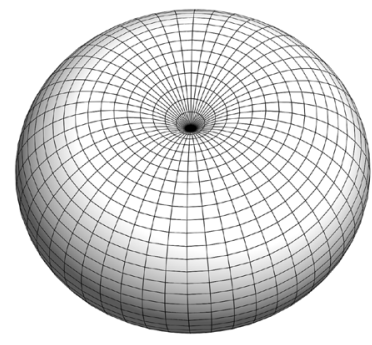

(b)

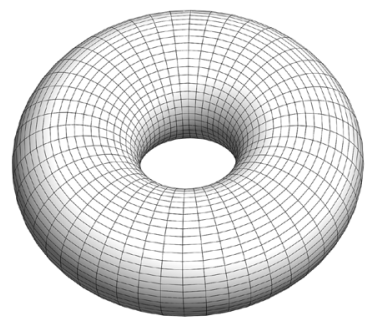

(c)

Figure 2. Vector field on sphere and torus: (a) A Singularity of the field on a sphere; (b) The same field on a sphere with a blind incurvation; (c) Torus, the field without singularities. 
field [9] [10] [12] [14]. Each cell of the field has a number of its edges corresponding to the number of neighboring cells. There are only five homogenous discrete fields on the sphere corresponding to five regular polyhedrons, Platonic bodies. During synchronous cleavage divisions, only the first four blastomeres can create a homogeneous field on the embryo surface (a cleaving embryo is considered as topological polyhedron, not geometrical one [9] [10]. After third division (8 blastomeres) the field pattern of cellular contacts on the embryo surface becomes topologically inhomogeneous inevitably that means the symmetry breaking of the discrete cell field. In hexagonal cell network, presence of cells with another number of sides (for example, with 5 or 7 sides) can be considered as a topological dislocation of homogeneous graph cells [28] (Figure 3(a), Figure 3(b)). This means that the symmetry of the field is breaking, and this symmetry breaking is a consequence of Euler's theorem on polyhedra [10] [12]. The number of adjacent cells for any cell of the discrete cell field is the local order of the field, and the general number of vertices, edges and faces of the given graph is the integral order of the field. So a discrete field on the spherical surface of a cleaving embryo inevitably has topological singularities, and the local singularities determine the integral pattern of a developing organism. Gauss-Bonnet theorem defines a discrete cell curvature, Gauss curvature (see [9] [10]). If the sum of adjacent faces is more than 6, the curvature of the face becomes negative. It was proposed a causal relation between cell negative curvature, which leads to incurvation (invagination) and following morphogenetic cell movement during gastrulation [9] [11] [12] [16]. According to the Gauss-Bonnet theorem, an increase of curvature at some location has to be counterbalanced by a corresponding decrease of curvature at another location [16]. At least in some animals, the localization of invagination (or immigration, ingression) during gastrulation coincides with the singularity of negative curvature i.e., with the highest value of the cell field on vegetal hemisphere of embryos, whereas cells of positive curvature are present near animal pole [9] [10] [12]. Figure 3(c), Figure 3(d) demonstrates topological inhomogeneities of discrete fields of cell contacts on the surface of animal and vegetal hemispheres of a cleaving crustacean embryo. Other examples of negative curvature singularities on vegetal surface of cleaving embryos in various animals were shown earlier [9] [11]. The appearance of non-hexagonal faces is inevitable since a sphere cannot be covered by hexagons alone. Cells with a negative and positive curvature inevitably emerge on the surface of an embryo, and the negative curvature determines the localization of invagination or immigration during gastrulation. The localization of the singularities of negative curvature on vegetal surface of these embryos coincides with the site of cell ingression during the following gastrulation process, whereas cells of positive curvature are present on the animal pole surface. Thus, gastrulation may be related to an inevitable emergence of discrete curvature that directs development in three-dimensional space.

Gastrulation is the first spherical surgery in development, a topological catastrophe. The completion of gastrulation is topological transition from the sphere with singularity (singularities) to the torus, which is a topologically stable object with homogeneous discrete field, without singularities [9] [10] [12] (Figure 2). So a postgastrulation embryo or larva with through intestine tube is a topologically stable object. According to L. Wolpert, "the most important time in your life is not birth, marriage, or death, but gastrulation" (cited after [16] [27] [29]). Gastrulation is a way of topological stabilization in embryogenesis; consequently, gastrulation is indeed a very important event in our life.

Gastrulation is an extensive rearrangement of cells in an embryo. In embryos, cell migration pathways during gastrulation and other morphogenetic processes [27] visualize the morphogenetic vector fields with singular points. Thus, the breaking of spatial symmetry of preexisting pattern is inevitable and plays a critical role in early development.

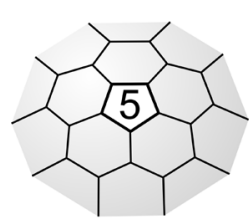

(a)

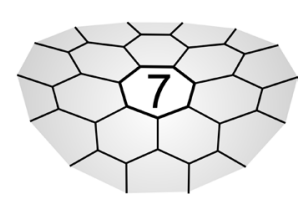

(b)

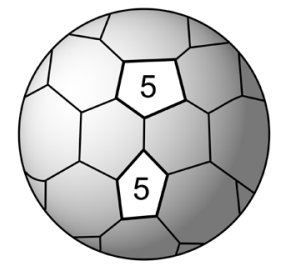

(c)

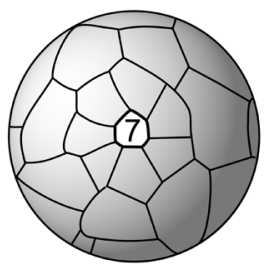

(d)

Figure 3. Topological inhomogeneities of cell discrete fields fields: (a) The cell of positive curvature among hexagonal cells; (b) The cell of negative curvature; (c) Two cells of positive curvature on animal hemisphere of a crustacean embryo; (d) The cell of negative curvature on vegetal hemisphere of the same embryo (after [10], modified). 


\section{Topological Surgeries in Metazoan Evolution and Development}

In metazoan evolution, appearance of the epithelial tissue was one of the most significant innovations [30] [31]. During metazoan development, epithelia determine the increasing complexity of the body changing their local curvature [16]. Such epithelial morphogenetic movements as the folding, spreading, budding, cavitation, and delamination produce reorganization of whole body plan [30]. Epithelial cells form cohesive cell sheets; the structural and functional connectivity of an epithelial layer during epithelial morphogenesis is ensured by the system of specialized intercellular contacts that integrate cells into a united morphological and functional entity [30] [31].

To translate traditional morphological descriptions of a metazoan morphogenesis into topological language the external shape of an organism is modeled as a set of smooth, closed, orientable surfaces formed by epithelial layers neglecting the thickness of the cellular layers [7] [8] [12] [13] [15] [16]. The establishment of apical-basal polarity of epithelial cells generates two surfaces of an epithelium with different components and functions [32] [33]. Thus, epithelial cell layers are characterized by connectivity, closeness of an intact surface and apical-basal polarity, anisotropy.

Metazoan morphogenesis may be represented as topological modification(s) of epithelial surfaces of spherical or toroidal shape. According to the theorem of elementary topology, any closed surface in three-dimensional space is homeomorphic (topologically equivalent) to the sphere with a certain number (p) of handles [8] [9] [11]-[14]. The sphere with $p$ handles sets a class of homeomorphic surfaces of the genus $p$. If there are no topological surgeries, such as breaking (cutting) and gluing of epithelial sheets, the genus of the surface $(p)$ is a topological invariant, and any geometrical deformations such as surface curvature, linear and angle values are not essential. The closed surfaces of the genus $p=0$ (sphere), $p=1$ (torus), $p=2$ (double torus, "pretzel”) and so on give a topological classification. The differences between these surfaces have a topological, qualitative nature [3].

Topological handles in biological objects are usually realized as channels (or canals, holes), for example, the digestive tube. We consider only epithelized through channels as topological handles. Repeated addition of "handles" or creation of "holes", "tunnels" has led to formation of double, triple, and higher order toroids [16]. An adult metazoan organism topologically is a sphere with a set of handles. Metazoan morphogenesis may be represented as topological modification(s) of closed epithelial surfaces.

Topological transitions in metazoan development occur due to topological surgeries realized by "cutting” and "gluing” of epithelial sheets [7] [8], as "self-wounding and healing” [16]. Local topological surgeries in epithelial morphogenesis involve complex processes at the cellular level: sheet disintegration followed by cell adhesion and cytoskeletal cooperation giving rise to a newly formed cell sheet (or sheets).

We can see two kinds of topological surgeries of epithelial surfaces in biological morphogenesis. Spherical surgeries change the genus of the surface. Another kind of topological surgeries changes the connectivity of epithelial layers by the separation of additional closed epithelial surfaces from preexisting ones or by the fusion of preexisting layers. The developmental modifications of connectivity do not change the topological pattern of the outer surface of animal body, so only the spherical surgeries changing the genus of the surface are considered here.

Topological patterns in metazoan evolution, from sponges to chordates, were analyzed using the genus of the surface as a topological invariant. We considered here (Figure 4) only basic topological archetypes of spatial organization for each taxon, neglecting variations and modifications inside the taxon. We used the interpretation of metazoan phylogeny, which consider three major clades of Bilateria: Deuterostomia, Lophotrochozoa, and Ecdysozoa (see, e.g., [34]).

In evolution of Metazoa we can find several topological transitions [12] [13]. In solitary cnidarians and such lower Bilateria as Acoelomorpha with a blind gut the kind of the surface is $p=0$. The first important topological surgery in evolution of Bilateria (Triploblastica) is the appearance of a through intestinal tube instead of a blind gut. Among Bilateria, animals of many phyla have the stable topological pattern with the through digestive tube without any other through channels, and their external surface is topologically homeomorphic to the torus, $p=1$ (Figure 4, Figure 5(a)). The appearance of a through gut is a topological innovation of great evolutionary importance resulting in the differentiation of digestive system and better utilization of nutrients from the external medium. Topologically the digestive tube is outer surface of an organism as well as ectodermal surface of the organism; both ecto- and entodermal epithelia form united continuous surface, the outer surface of an organism. 


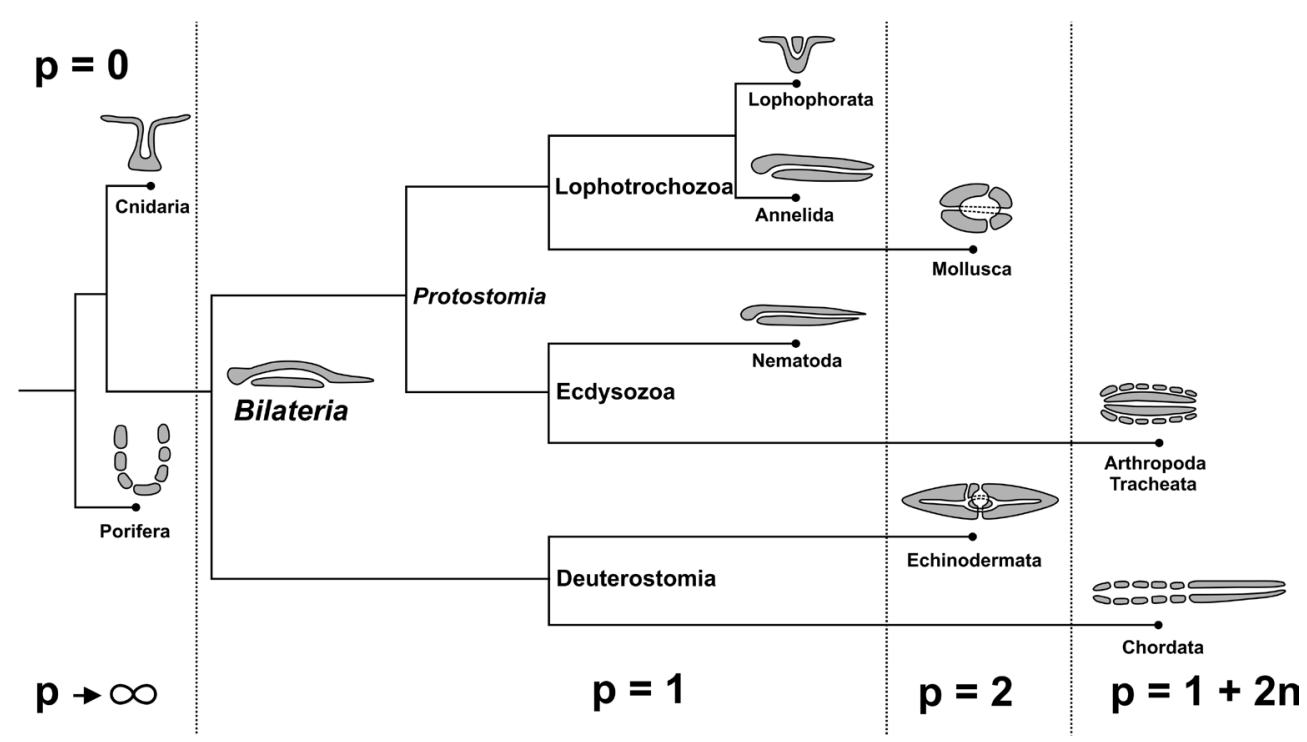

Figure 4. The scheme of main topological body patterns in Metazoa using genus of surface $(p)$.

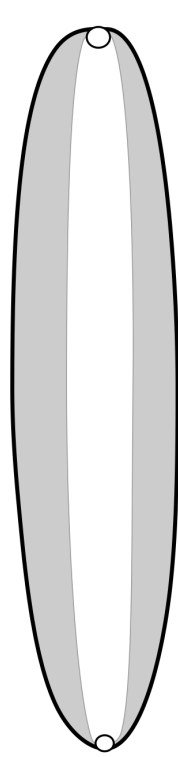

(a)

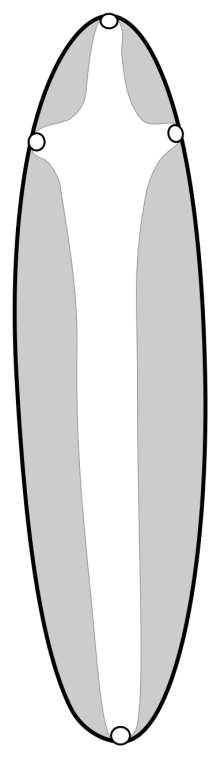

(b)

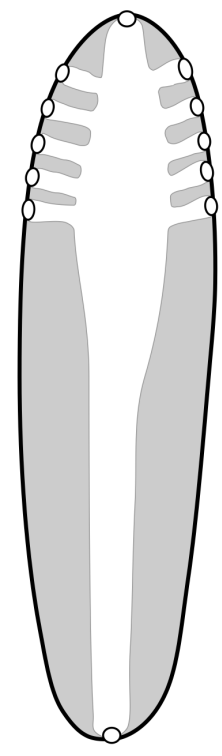

(c)

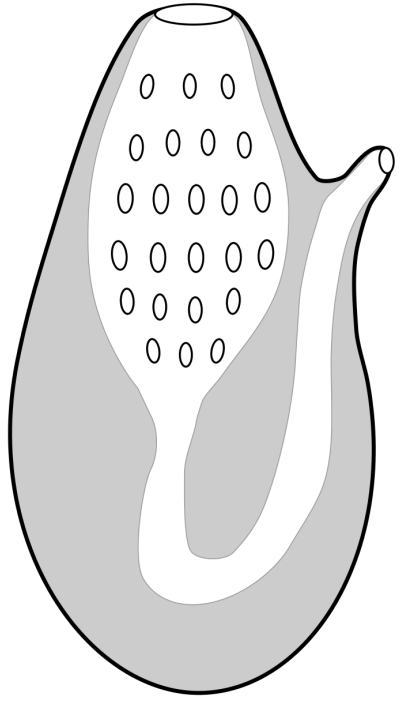

(d)

Figure 5. Topological patterns in some hemichordates and lower chordates: (a) A simple digective tube with oral and anal openinhs in most invertebrates; (b) The digestive canal with two additional lateral openings (Pterobranchia, Appendicularia); (c) The digestive tube with several lateral slit openings (Enteropneusta and chordate archetype; (d) The digestive tube with numerous pharyngeal openings (Urochordata) after [13].

Subsequent evolution has led to the formation of the through channels of the coelomic and respiratory systems. The surface of an organism having another additional through channel besides the digestive tube is a surface of genus 2, which is topologically equivalent to a sphere with two handles, double torus or "pretzel". For example, echinoderms exhibit the second through channel, namely the ambulacral system. The spatial organization of the circular ambulacral system connected with the external medium by a canal is topologically equivalent to a through channel [12] [13]. So an archetype of the topological design of an adult echinoderm body can be represented by the double torus. In the molluscan archetype, the second through channel of the coelomic system was the result of fusion of paired coeloms via coelomoducts. Acquisition of the surface of the genus $p=2$ may 
be considered as a side branch in the evolution of both Deuterostomia (in Echinodermata) and Protostomia (in some Lophotrochzoa).

The next evolutionary level of topological design in animals was attained through the development of the respiratory system. The system of paired tracheal tubules with lateral connections (of ectodermal origin) in Tracheata (higher terrestrial arthropods) or paired gill slits (of entodermal origin) in a chordate archetype evolved (Figure 5(c)). Accordingly, a parallelism of topological and functional innovations in the evolution of deuterostomes and of some protostomes (among Ecdysozoa) is obvious as a transition to the surface of the genus $p=1+$ $2 n$, by adding an even number of channels (handles) of the respiratory system. The creation of through respiratory system is the topological modification of the surface, which results in better utilization of oxygen from outside medium flowing through the organism and more intensive metabolism. Through channels of the respiratory system are filled with external medium and may be considered as an outer surface of the animal body.

There are various evolutionary modifications of the archetypical body plan in many phyla due to additional topological surgeries. Colonial cnidarians are "multi-oral" organisms, i.e., multiple tori. In Spongia and in some Cnidaria, an increase of genus $p$ up to very high values and the shaping of topologically complicated fractal systems are evident. In some scyphomedusae and creeping ctenophores branching and anastomosing canals of gastro-vascular system result in the increasing of genus $p$ up to indefinite high values. In some flat worms (Platyhelminthes) complicated topological and fractal patterns have arisen on the base of the branched gut and excretory system [13] [14].

Hemichordata have a through gut and several pairs of gill slits $(p=1+2 n)$ similar to chordate archetype; in some Appendicularia, the digestive canal has two additional lateral openings (Figure 5(b), Figure 5(c)). Adult mammals and some other vertebrates (Chordata, Vertebrata) also exhibit a triple-torus pattern because their nostrils are connected to the gastrointestinal tract [16]; gill slits in vertebrates are transient structures disappearing during embryogenesis. In birds, the genus of the body surface attains very high values because of numerous inner anastomoses in their bronchial system, which forms the bronchiolar network [13]. In ascidians (Chordata: Urochordata), the body surface is a high-order torus due to the presence of numerous gill openings (Figure 5(d)).

Thus, topological transformations of the body surface during metazoan evolution resulted in better distribution of flows to and from the external medium, used as the source of nutrients and oxygen and the sink of excreta, so ensuring greater metabolic intensity and better adaptation of organism to the environment.

An analysis of topological organization of the closed epithelial layer that covers the outer body surface as a continuous envelope is applicable to the topological organization of the body surface in metazoan development [12]-[14] [16]. During embryonic development, the surface of most metazoan animals undergoes sequential spherical surgeries, which change the topological genus of the surface, and developmental morphogenesis may be represented by series of topological surgeries of the closed body surface. Local topological surgeries inevitably lead to global topological modifications of biological forms, as the genus of the surface is a global property.

No modification is observed in topology of the body surface during embryonic development of those animals which in adult state have the zero genus of the surface as some solitary cnidarians and Acoelomorpha [12] [13] [16].

The surfaces of an egg, blastula as well as early gastrula in metazoan animals are surfaces of the genus 0 , homeomorphic to a sphere (Figure 6(a)). In most Metazoa, the development of an organism beginning from the surface of the genus 0 leads to an adult animal which genus of the surface differs from zero. A transition from blind archenteron to through digestive tube is realized during gastrulation by the formation of another opening besides the blastopore (a primary mouth); the last becomes the definitive oral opening in Protostomia, or anal opening in Deuterostomia. The epithelial surface of an embryo after gastrulation or a larva possessing a through intestine tube (having both oral and anal openings) is a surface of genus 1, topologically equivalent to a sphere with one handle or to a torus Figure 6(b)). In animals having in the adult state the genus of the body surface $p=$ 1 (for instance, in round and annelid worms), only the transition $p=0 \rightarrow p=1$ occurs during development.

The surface of an organism having another additional through channel besides the digestive tube is the surface of genus 2, topologically equivalent to a sphere with two handles, double torus or "pretzel". For example, sea urchins and other echinoderms exhibit a second through channel, formed by the ambulacral system (Figure 6(c)). In sea urchins, the spatial organization of the circular ambulacral system connected with the external medium by a canal is topologically equivalent to a through channel (see [12] [13]). There are two topological transformations: $p=0 \rightarrow p=1 \rightarrow p=2$ in development of sea urchins and other echinoderms (as well as in molluscan development). 

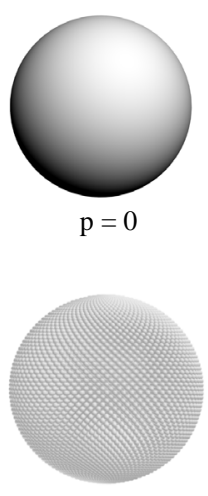

(a)

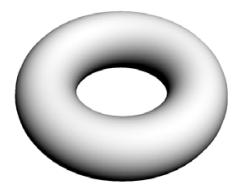

$\mathrm{p}=1$

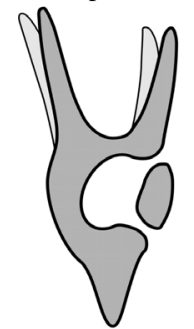

(b)

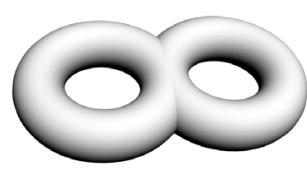

$\mathrm{p}=2$

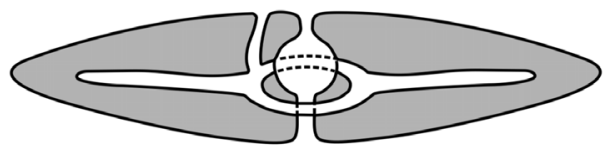

(c)

Figure 6. Topological patterns in sea urchin development: (a) An egg; (b) A larva (pluteus); (c) Adult animal.

During embryonic development of the Tracheata (among Arthropoda) and some representatives of Hemichordata, the following transformations of the genus of the body surface take place: $p=0 \rightarrow p=1 \rightarrow p=1+2 n$ [12] [13].

The detailed analysis of topological form dynamics in ontogenesis may provide additional possibilities for metazoan description and classification. For example, we have considered the different dynamics of numerical characteristics of four types of channels (gut, ambulacral system, hydropore, and pores through the madrepore plate) during embryogenesis, metamorphosis and in adult animals in different classes of Echinodermata [12].

Topological surgeries of another kind modify connectivity of germ layers and result in the separation of a set of additional closed epithelial surfaces from preexisting ones, for example, enterocoelic formation of mesoderm in Deuterostomia, neurulation and eye cap formation in Chordata and so on [7] [8]. The topological surgeries of connectivity do not change the genus of the outer surface. The spatial organization of evolutionarily advanced animals may be represented topologically as an outer epithelial envelope of a certain genus $p$ embracing a number of inner closed epithelial surfaces embedded inside the outer envelope.

At cell level, topological approach was used to describe cell membrane system and membrane dynamics [12] [14] [16] [35]. Cell membrane systems as well as epithelia can be described as closed and orientable surfaces. Cell membrane systems undergo topological surgeries of the connectivity with perpetual separations of additional closed membrane surfaces from pre-existing ones and, inversely, fusions of membrane surfaces. These topological surgeries modifying the spatial organization of cell membrane system are the dynamic expression of cell functions.

The spatial organization of a eukaryotic cell may be represented topologically as a number of inner closed membrane surfaces of cell organelles embedded inside the outer cell membrane. Following Y. Tashiro [32] we introduce the enclosing (embedding) number to numerically evaluate membrane surfaces sequentially enclosed one into another (as Russian dolls). The enclosing number for outer cell membrane (embedded inside an external space) is 1 , the enclosing number for most intracellular membranes (outer mitochondrial membranes, membranes of Golgi systems, nuclear envelope) is 2; the enclosing number of inner mitochondrial membranes is 3 (Figure 7). The nuclear envelope in eukaryotic cells is an embedded intracellular membrane system with multiple pores, or a multiple toroid. Plastids in most plants have the outer membrane of the enclosing number 2, the enclosing number of the inner membrane is 3 , and thylacoid membranes have the enclosing number 4 . In some unicellular organisms, plastids have more membranes [36]; these membranes are sequentially embedded one into another, so the enclosing number of thylacoid membranes becomes 5 or 6 .

\section{Conclusions}

Topological singularities inevitably emerging and transforming in biological morphogenesis disrupt preexisting symmetry pattern. The symmetry of a metazoan body, including the scale symmetry of fractal structures, the translational symmetry of metamerism and other variants of morphofunctional iterations is an efficient means of 


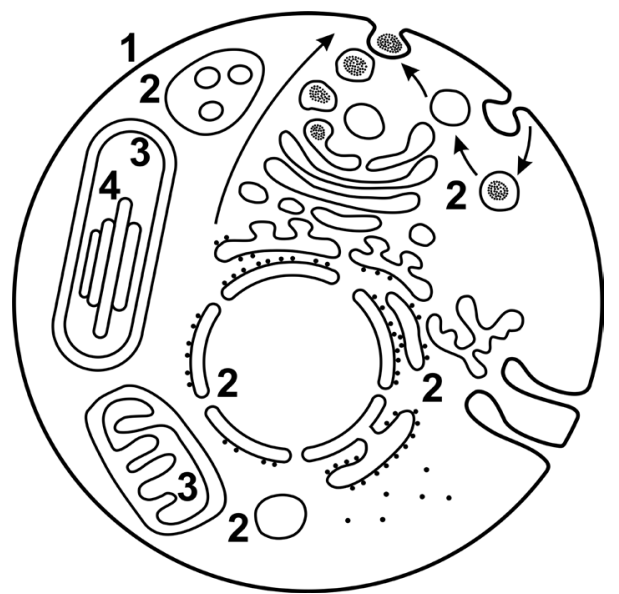

Figure 7. Topological model of membrane systems of a eukaryotic cell; membrane enclosing numbers are indicated.

modular morphogenesis. Evolutionary developmental biology includes the concept of modularity, according to which ontogenesis consists of relatively independent processes, dissociable modules; structural elements of the developing organism may also be presented as discrete dissociable units. Dissociation of developmental modules and their recombination leads to evolutionary transformations with duplications and subsequent divergence, insertions, deletions and substitutions (see [37] [38]). This kind of evolutionary transformations was called "evolutionary cut and paste" [37]. Evolutionary cutting and pasting are the same as topological breaking (cutting) and gluing. Essentially, "cut and paste" approach is topological one.

The architecture of gene regulatory networks is modular (see [38] [39]). In evolution, duplications of genes, gene clusters, modules of gene networks and whole genomes were revealed. The co-linearity phenomenon as a mapping of the linear arrangement of Hox-genes in their cluster (or clusters) in the chromosome (chromosomes) into the spatial and temporal sequence of gene expression along a body axis also can be interpreted in terms of topology as genotype-phenotype mapping [15]. Hox-genes are known as genes-architects determining the body plan in Bilateria [27]. The deletion of a Hox-gene or a disturbance of the gene order in the Hox-cluster can cause large-scale macro-evolutionary transformation in body morphology (see, e.g., [40] [41]). Thus, topological rearrangement by cutting (breaking) and pasting (glueing) can be found at all levels of biological organization.

Symmetry breaking during oogenesis and early development is a fundamental event establishing the main axial coordinates of a future organism, while in later development the scale of symmetry breaking is decreasing. Topological rehandlings are regular processes in ontogeny and in phylogeny and involve the emergence of discontinuities [1]. Topological singularities inevitably emerge and transform in biological morphogenesis. The inevitability of egg cell polarization as the emergence of the topological singularity of the vector field on spheres follows from the Poincaré-Hopf theorem. The presence of a fixed point on the cortical sphere of an egg cell is inevitable for ooplasmic segregation according to Brouwer's theorem. Zygote division creates pattern of contacts on the surface of an embryo, i.e., a discrete morphogenetic field with inevitable topological singularities. According to the Gauss-Bonnet theorem, cells with a negative or positive curvature inevitably emerge on the surface of an embryo, and the negative curvature can determine the location of invagination during gastrulation. Gastrulation is a topological transition from the sphere with heterogeneity and singularity of the field to the torus with a homogeneous field.

Subsequent metazoan morphogenesis may be represented as a succession of spherical surgeries of closed orientable epithelial surfaces. The local topological surgery leads to the global topological modification of biological forms. The epithelial surface is an interface between the internal medium of an organism and the outside environmental medium; topological and fractal transformations during metazoan evolution and development increases this interface, ensuring metabolic efficiency and better adaptation of organism to the environment.

The course and outcome of biological evolution is strongly influenced by constraints; evolution and development result in discontinuities and directionality of morphological transformations [42]. The inevitable topological dependence and constraints are integrated, fitted into genetically determined processes of biological mor- 
phogenesis, which cannot be independent of physical and topological organization of our space [14] [15]. Topological methodology reveals a certain set of topological rules that constrain and direct biological morphogenesis [9] [14].

It was supposed that some biological forms, such as branching structures, are the most functional design; these forms "are topological attractors that evolution cannot avoid" [43]. Just as branching structures, toroid forms can be considered as functionally optimized biological design and topological attractors in biological morphogenesis.

\section{References}

[1] Bouligand, Y. (1996) Morphological Singulatities and Macroevolution. Memorie della Societa Italiana di Scienze Naturali e de Museo Civico di Storia Naturale di Milano, 27, 89-94.

[2] Thom, R. (1969) Topological Models in Biology. Topology, 8, 313-335. http://dx.doi.org/10.1016/0040-9383(69)90018-4

[3] Thom, R. (1996) Qualitative and Quantitative in Evolutionary Theory with Some Thoughts on Aristotelian Biology. Memorie della Societa Italiana di Scienze Naturali e de Museo Civico di Storia Naturale di Milano, 27, 115-117.

[4] Thom, R. (1997) Structural Stability and Morphogenesis: An Outline of a General Theory of Models. Addison-Wesley Publishing Co., New York.

[5] Wasserman, S.A. and Cozzarelli, N.R. (1986) Biochemical Topology: Applications to DNA Recombination and Replication. Science, 232, 951-960. http://dx.doi.org/10.1126/science.3010458

[6] Monastyrsky, M.I. (2007) Topology in Molecular Biology. Springer-Verlag, Berlin. http://dx.doi.org/10.1007/978-3-540-49858-2

[7] Maresin, V.M. and Presnov, E.V. (1985) Topological Approach to Embryogenesis. Journal of Theoretical Biology, 114, 387-398. http://dx.doi.org/10.1016/S0022-5193(85)80174-0

[8] Presnov, E.V., Malyghin, S.N. and Isaeva, V.V. (1988) Topological and Thermodynamic Structure of Morphogenesis. In: Lamprecht, I. and Zotin, A.I., Eds., Thermodynamics and Pattern Formation in Biology, Walter de Gruyter, Berlin, 337-370.

[9] Presnov, E., Isaeva, V. and Kasyanov N. (2010) Topological Determination of Early Morphogenesis in Metazoa. Theory in Bioscience, 129, 259-270. http://dx.doi.org/10.1007/s12064-010-0103-y

[10] Presnov, E., Isaeva, V. and Kasyanov, N. (2014) Topological Invariance in Biological Development. Axiomathes, 24, 117-135. http://dx.doi.org/10.1007/s10516-013-9216-5

[11] Presnov, E.V. and Isaeva, V.V. (1991) Local and Global Aspects of Biological Morphogenesis. Speculations in Science and Technology, 14, 68-75.

[12] Presnov, E.V. and Isaeva, V.V. (1996) Topological Classification: Onto- and Phylogenesis. Memorie della Societa Italiana di Scienze Naturali e de Museo Civico di Storia Naturale di Milano, 27, 89-94.

[13] Isaeva, V., Presnov, E. and Chernyshev, A. (2006) Topological Patterns in Metazoan Evolution and Development. Bulletin of Mathematical Biology, 68, 2053-2067. http://dx.doi.org/10.1007/s11538-006-9063-2

[14] Isaeva, V.V., Kasyanov, N.V. and Presnov, E.V. (2008) Analysis Situs of Spatial-Temporal Architecture in Biological Morphogenesis. In: Kelly, J.T., Ed., Progress in Mathematical Biology Research, Nova Science Publishers, New York, 141-189.

[15] Isaeva, V.V., Kasyanov, N.V. and Presnov, E.V. (2012) Topological Singularities and Symmetry Breaking in Development. Biosystems, 109, 280-298. http://dx.doi.org/10.1016/j.biosystems.2012.05.004

[16] Jockush, H. and Dress, A. (2003) From Sphere to Torus: A Topological View of the Metazoan Body Plan. Bulletin of Mathematical Biology, 65, 57-65. http://dx.doi.org/10.1006/bulm.2002.0319

[17] Greenblum, S., Turnbaugh, P.J. and Borenstein, E. (2012) Metagenomic Systems Biology of the Human Gut Microbiome Reveals Topological Shifts Associated with Obesity and Inflammatory Bowel Disease. Proceedings of the National Academy of Sciences of the United States of America, 109, 594-599. http://dx.doi.org/10.1073/pnas.1116053109

[18] Dabaghian, Y., Memoli, F., Frank, L. and Carlsson, G. (2012) A Topological Paradigm for Hippocampal Spatial Map Formation Using Persistent Homology. PLoS Computational Biology, 8.

[19] Niizato, T., Murakami, H. and Gunji, Y.P. (2014) Emergence of the Scale-Invariant Proportion in a Flock from the Metric-Topological Interaction. Biosystems, 119, 62-68. http://dx.doi.org/10.1016/j.biosystems.2014.03.001

[20] Li, R. and Bowerman, B. (2010) Symmetry Breaking in Biology. Cold Spring Harbor Perspectives in Biology, 2, a003475.

[21] Nüsslein-Volhard, C. (1991) Determination of the Embryonic Axes of Drosophila. Development. Supplement, 1, 1-10. 
[22] Mullins, R.D. (2010) Cytoskeletal Mechanisms for Breaking Cell Symmetry. Cold Spring Harbor Perspectives in Biology, 2, a003392.

[23] Nuccitelli, R. (1984) The Involvement of Transcellular Ion Currents and Electric Fields in Pattern Formation. In: Malacinski, G.M. and Bryant, S.V., Eds., Pattern Formation: A Primer in Developmental Biology, MacMillan, London, 23-46.

[24] Vacquer, V.D. (1981) Dynamic Changes of the Egg Cortex. Developmental Biology, 84, 1-26. http://dx.doi.org/10.1016/0012-1606(81)90366-3

[25] Kirschner, M.W. and Gerhart, J.C. (2005) The Plausibility of Life. Yale University Press, Yale, New Haven.

[26] Gilbert, S.F. (2000) Developmental Biology. Sinauer Associates, Inc., Sunderland.

[27] Kline, D. and Nuccitelli, R. (1985) The Wave of Activation Current in the Xenopus Egg. Developmental Biology, 111, 471-487. http://dx.doi.org/10.1016/0012-1606(85)90499-3

[28] Rivier, N., Miri, M.F. and Oguey, C. (2005) Plasticity and Topological Defects in Cellular Structures: Extra Matter, Folds and Crab Moulting. Colloids and Surfaces A, 263, 39-45. http://dx.doi.org/10.1016/j.colsurfa.2005.01.027

[29] Vogel, G. (2000) Tracking the Movements that Shape an Embryo. Science, 288, 86-87. http://dx.doi.org/10.1126/science.288.5463.86

[30] Kolega, J. (1986) The Cellular Basis of Epithelial Morphogenesis. In: Browder, W., Ed., Developmental Biology, Vol. 2, Plenum Press, New York, 103-143.

[31] Zallen, J.A. (2007) Planar Polarity and Tissue Morphogenesis. Cell, 129, 1051-1063. http://dx.doi.org/10.1016/j.cell.2007.05.050

[32] Nelson, C.M. (2009) Geometric Control of Tissue Morphogenesis. Biochimica et Biophysica Acta-Molecular and Cell Research, 1793, 903-910.

[33] Vladar, E.K., Antic, D. and Axelrod, J.D. (2009) Planar Cell Polarity Signaling: The Developing Cell’s Compass. Cold Spring Harbor Perspectives in Biology, 1, a002964.

[34] Halanych, K.M. (2004) The New View of Animal Phylogeny. Annual Review of Ecology, Evolution and Systematics, 35, 229-256. http://dx.doi.org/10.1146/annurev.ecolsys.35.112202.130124

[35] Tashiro, Y. (1983) Subcellular Compartments and Protein Topogenesis. Cell Structure and Function, 8, 91-107. http://dx.doi.org/10.1247/csf.8.91

[36] Whatley, J.M. and Whatley, E.R. (1984) Evolutionary Aspects of the Eukaryotic Cell and Its Organelles. In: Lindskens, H.F. and Heslop-Harrrison, J., Eds., Cellular Interactions, Springer Verlag, Berlin, 18-58.

[37] Shubin, N.H. (1998) Vertebrate Palaeontology: Evolutionary Cut and Paste. Nature, 394, 12-13. http://dx.doi.org/10.1038/27755

[38] Raff, R.A. and Sly, B.J. (2000) Modularity and Dissociation in the Evolution of Gene Expression Territories in Development. Evolution and Development, 2, 102-113. http://dx.doi.org/10.1046/j.1525-142x.2000.00035.x

[39] Peter, I.S. and Davidson, E.H. (2011) Evolution of Gene Regulatory Networks Controlling Body Plan Development. Cell, 144, 970-985. http://dx.doi.org/10.1016/j.cell.2011.02.017

[40] Minelli, A. (2003) The Development of Animal Form: Ontogeny, Morphology, and Evolution. Cambridge University Press, Cambridge. http://dx.doi.org/10.1017/CBO9780511541476

[41] Deutsch, J.S. and Mouchel-Vielh, E. (2003) Hox Genes and the Crustacean Body Plan. BioEssays, 25, 878-887. http://dx.doi.org/10.1002/bies.10319

[42] Sará, M. (1999) New Perspectives on the Role of Constraints in Evolution. Rivista Biologica/Biological Forum, 92, 29-52.

[43] Thomas, R.D.K. and Reif, W.E. (1993) The Skeleton Space: A Finite Set of Organic Designs. Evolution, 47, 341-360. http://dx.doi.org/10.2307/2410056 
Scientific Research Publishing (SCIRP) is one of the largest Open Access journal publishers. It is currently publishing more than 200 open access, online, peer-reviewed journals covering a wide range of academic disciplines. SCIRP serves the worldwide academic communities and contributes to the progress and application of science with its publication.

Other selected journals from SCIRP are listed as below. Submit your manuscript to us via either submit@scirp.org or Online Submission Portal.
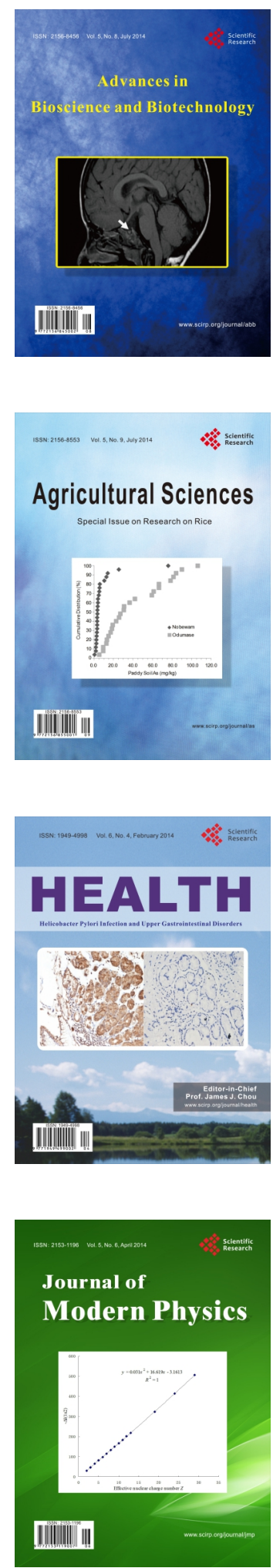
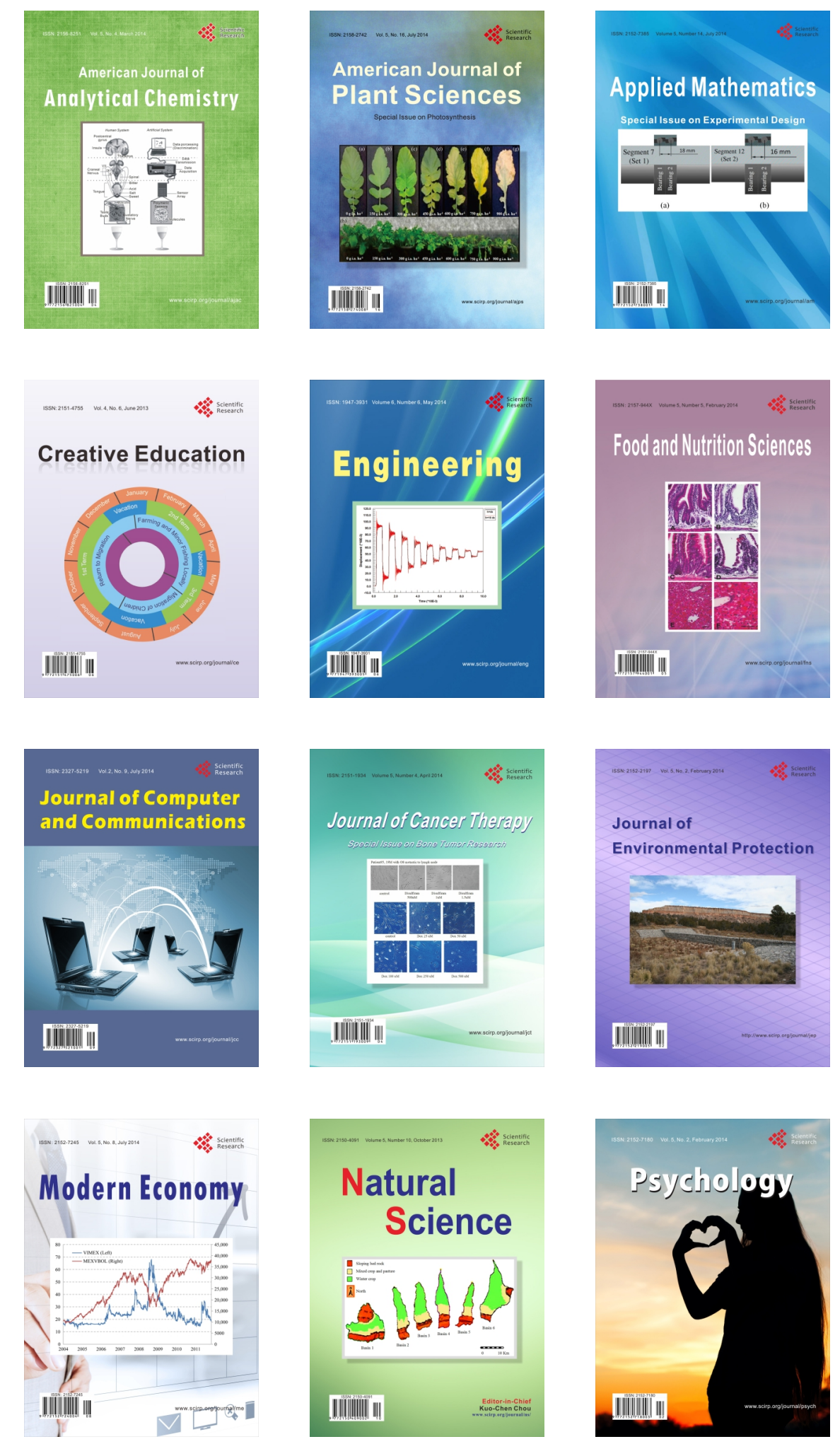